\title{
College admissions with stable score-limits
}

\author{
Péter Biró* \\ Institute of Economics, Research Centre for Economic and Regional Studies, \\ Hungarian Academy of Sciences, and \\ Corvinus University of Budapest, \\ Department of Operations Research and Actuarial Sciences \\ H-1112, Budaörsi út 45, Budapest, Hungary \\ Email: peter.biro@krtk.mta.hu. \\ Sofya Kiselgof ${ }^{\dagger}$ \\ Laboratory of Decision Choice and Analysis (DecAn), \\ NRU Higher School of Economics, Moscow, Russia \\ Email: skiselgof@hse.ru.
}

\begin{abstract}
A common feature of the Hungarian, Irish, Spanish and Turkish higher education admission systems is that the students apply for programmes and are ranked according to their scores. Students who apply for a programme with the same score are tied. Ties are broken by lottery in Ireland, by objective factors in Turkey (such as date of birth) and other precisely defined rules in Spain. In Hungary, however, an equal treatment policy is used, students applying for a programme with the same score are all accepted or rejected together. In such a situation there is only one decision to make, whether or not to admit the last group of applicants with the same score who are at the boundary of the quota. Both concepts can be described in terms of stable score-limits. The strict rejection of the last group with whom a quota would be violated corresponds to the concept of H-stable (i.e. higher-stable) score-limits that is currently used in Hungary. We call the other solutions based on the less strict admission policy as L-stable (i.e. lower-stable) score-limits. We show that the natural extensions of the Gale-Shapley algorithms produce stable score-limits, moreover, the applicant-oriented versions result in the lowest score-limits (thus optimal for students) and the college-oriented versions result in the highest score-limits with regard to each concept. When comparing the applicant-optimal H-stable and L-stable score-limits we prove that the former limits are always higher for every college. Furthermore, these two solutions provide upper and lower boundaries for any solution arising from a tie-breaking strategy. Finally we show that both the H-stable and the L-stable applicant-proposing score-limit algorithms are manipulable.
\end{abstract}

\section{Keywords: college admissions, stable matching, mechanism design JEL classification: C78, I21}

${ }^{*}$ This work was supported by OTKA grant K69027 and by the Hungarian Academy of Sciences under its Momemtum Programme (LD-004/2010).

${ }^{\dagger}$ The study was implemented in the framework of the Basic Research Program at the National Research University Higher School of Economics in 2012-2013 


\section{Introduction}

Gale and Shapley [14] introduced a model and solution concept to solve the college admissions problem fifty years ago ${ }^{1}$. In their model they suppose that the students submit preference lists containing the colleges they apply to, and each college ranks their applicants in a strict order and also provides an upper quota. Based on the submitted preferences a central body computes a fair solution. The fairness criterion they propose is stability, which essentially means that if an application is rejected then it must be the case that the college has filled its quota with applicants better than the applicants concerned. They gave an efficient method to find a stable matching and they proved that it is actually optimal for the students, in the sense that no student can be admitted to a better college in another stable matching. The Gale-Shapley algorithm has linear time implementation (see e.g. Knuth [19]), which means that the running time of the algorithm is proportional to the number of applications. Another attractive property of this matching mechanism, proved by Roth [22], is that it is strategy-proof for the students, i.e., no student can be admitted to any better college by submitting false preferences.

Later, it turned out (Roth [24]) that the algorithm proposed by Gale and Shapley had already been implemented in 1952 in the National Resident Matching Program and has been used since to coordinate junior doctor recruitment in the US. Moreover, the very same method has been implemented recently in the Boston [4] and New York [3] high school matching programs. However, college admissions are still organized in a completely decentralized way in the US, with all its flaws, that is unraveling through early admissions and coordination problems caused by too many or not enough students admitted. See some representative stories on American college admissions practices in the blog of $\mathrm{Al}$ Roth [34].

There are many other countries where higher education admissions are more regulated, but not yet centralized. In Russia, the common timetable of admissions prevents unraveling and the use of 'original documents' provided better coordination regarding the number of students admitted, but yet the solution is far from being optimal. ${ }^{2}$ In the UK, there is a common platform to manage the admissions by UCAS [35]. There are centralized admission framework, but the decisions and actions of the users (students and higher education institutions) are still decentralized.

Finally, there are some countries which do have centralized matching schemes for higher education admissions. In particular, there are scientific papers on the Chinese [27, 28], German [10, 26, 30], Hungarian [6, 7], Spanish [21] and Turkish [5] schemes. ${ }^{3}$

The Chinese higher education admissions system is certainly the largest in the world, with more than 20 million students enrolled in 2009 [28]. The system is based on a centralized exam, called the National College Entrance Examinations, which provide a score assigned to each student. This induces a ranking of the students by universities. The matching process (see [27]) is a kind of Boston mechanism with some extra tweaks that makes the system manipulable and controversial. The German clearinghouse for

\footnotetext{
${ }^{1}$ The 2012 Nobel-Prize in Economic Sciences has been awarded to Alvin Roth and Lloyd Shapley for the theory of stable allocations and the practice of market design.

${ }^{2}$ Each applicant applies to at most five universities, but does not inform universities about her preferences among them. Universities rank students using results of Unified State Exams. Two 'admission rounds' are organized that are similar to the first two steps of a deferred acceptance procedure. After the second step, universities that still have empty seats are allowed to organize additional admissions.

${ }^{3}$ However, we shall note that regrettably these scientific papers deal only with some special features of these systems (as we also do in this paper) so not all the aspects of these schemes are described. But luckily, there is a new European research network, called Matching in Practice [33], one of whose aim is to collect and describe current matching practices in Europe. So hopefully we will have a better picture and understanding on the current practices, at least in Europe.
} 
higher education admissions deals only with a small segment of subjects (about 13,000 students from the total 500,000, see [30]). The clearinghouse is a mixed system, in the first phase (for the best students) the Boston-mechanism is used and in the second phase the college-proposing Gale-Shapley, so the process is not incentive compatible for the group of best students $[10,26]$.

The Hungarian, Irish, Spanish and Turkish higher education matching schemes are all based on a centralized scoring system. The Irish system has not been described yet in a scientific paper to the best of our knowledge. ${ }^{4}$ In the other three countries students are assigned a score with regard to each programme they applied to, these scores coming mainly from their grades and entrance exams. The scores of a student may differ in two programmes, as when calculating the score of a student for a particular programme, only those subjects are considered which are relevant for that programme. The solution of the admission processes are represented by the so-called score-limits, which are referred to as 'base scores' in Turkey [5] and 'cutoff marks' in Spain. The score-limit of a programme means the lowest score that allows a student to be admitted to that programme. The scorelimits together with the preferences of the students naturally induce a matching, where each student is admitted to the first place on her list where she achieved the score-limit.

In Turkey [5] the ties are broken according to the date of birth of the students and the college-proposing Gale-Shapley algorithm is used. In Spain the scoring method is sufficient(the admission marks are from 5 to 14 with 3 decimal fractions, and some further priority rules are also used), so ties are very unlikely. They use the applicant-proposing Gale-Shapley algorithm with the special feature of limiting the length of the preference lists, a setting that creates strategic issues that were studied in detail by Romero-Medina [21] and Calsamiglia et al. [11].

In fact, in most applications where ties may occur, the programme coordinators break these ties. In the high school matching schemes in New York [3] and Boston [4] a lottery is used for breaking ties. However, this may lead to suboptimal solutions as Erdil and Erkin [12] point out, but according to the study by Abdulkadiroglu et al [1] this is the only way to keep the mechanism strategy-proof. In the Scottish Foundation Allocation Scheme [32], where the junior doctors are matched to hospitals, the organizers attempt to break the ties in such a way that in the resulting matching as many doctors are allocated as possible (see Irving and Manlove [17]). ${ }^{5}$

In contrast, in the Hungarian higher education admission scheme [31] the ties are not broken, therefore the students applying for a particular programme with equal scores are either all accepted or all rejected. We call this an equal treatment policy.

In particular, the ties are handled in the following way in Hungary. No quota may be violated, so the last group of students with the same score, with whom the quota would be exceeded, are all rejected. There is however an alternative policy, that could be followed where the quotas may be exceeded by the admission of the last group of students with the

\footnotetext{
${ }^{4}$ From the information published at the website of the Central Applications Office [29] it seems that the college-proposing Gale-Shapley algorithm is used in Ireland with some special features. One is that students can apply for 'level 8' and 'level $7 / 6$ ' courses simultaneously, and these applications are processed separately, so a student may receive more than one offer at a time. There are deadlines for accepting offers and if offers are rejected then further offers are made by the higher education institutions, so the mechanism is somewhat decentralized. The tie-breaking is based on 'random-numbers' assigned to students with regard to each programme they applied for, so the ties are broken differently for different programmes involving perhaps the same applicants.

${ }^{5}$ In SFAS [32], applicants are ranked by NHS Education for Scotland in a so-called master list, in order of score each applicant has a numerical score allocated partly on the basis of academic performance and partly as a result of the assessment of their application form. The range of possible scores (approximately 40 100) is much smaller than the number of applicants (around 750 each year), so there are ties of substantial length in the master list.
} 
same score, but only if there were unfilled places left otherwise.

As we will show in Section 3, both concepts can lead to matchings that satisfy special stability conditions based on score-limits that we formalize in Section 2. We refer to the first, more restrictive solution as H-stable (i.e., higher-stable) score-limits and we call the second, more permissive solution L-stable (i.e., lower-stable) score-limits. Note that these stable score-limit concepts generalize the original notion of stability by Gale and Shapley, since they are equivalent in the case of no tie. In Section 3, we show how one can extend the Gale-Shapley algorithm to find H-stable and L-stable score-limits. Moreover, in Section 4 we prove that the applicant-oriented versions provide the minimal stable score-limits (therefore they are the best possible solutions for the applicants), whilst the college-oriented versions provide maximal stable score-limits (therefore, they are the worst possible solutions for the applicants). We note that the above results are deducible from some general theorems on substitutable choice functions by Kelso and Crawford [18] and Roth [23], as it was very recently demonstrated by Fleiner and Jankó [13]. We describe these arguments in detail at the end of Section 4.

In Section 5 we show that comparing the H-stable and L-stable score-limits, the Lstable score-limits are more favorable for the applicants as they are lower. In particular, we show that no college can have a higher score-limit in the applicant-optimal L-stable solution than in the applicant-optimal H-stable solution (and the same applies for the applicantpessimal solutions produced by the college-oriented versions). Interestingly, we also show that the applicant-optimal solution produced after a tie-breaking is always between these two kinds of solutions. Therefore the matchings corresponding to the $\mathrm{H}$-stable and Lstable score-limits may provide upper and lower boundaries for every applicant regarding her match in a scheme which uses any kind of tie-breaking strategy. Finally, in Section 6 we give examples showing that neither the H-stable nor the L-stable version of the applicant-oriented score-limit algorithm is strategy-proof. We conclude in Section 7.

\section{The definition of stable score-limits}

Let $A=\left\{a_{1}, a_{2}, \ldots, a_{n}\right\}$ be the set of applicants and $C=\left\{c_{1}, c_{2}, \ldots, c_{m}\right\}$ be the set of colleges, where $q_{u}$ denotes the quota of college $c_{u}$. Let the ranking of the applicant $a_{i}$ be given by a preference list $P^{i}$, where $c_{v}>_{i} c_{u}$ denotes that $c_{v}$ precedes $c_{u}$ in the list, i.e. the applicant $a_{i}$ prefers $c_{v}$ to $c_{u}$. Let $s_{u}^{i}$ be $a_{i}$ 's final score at college $c_{u}$. Final scores are considered to be positive integers for all acceptable applicants. The students with scores below a common minimum threshold are rejected automatically (currently this minimum score is 240 in Hungary with a maximum score of 500, which applies for every study). In the model we use zero as a minimum threshold.

The score-limits of the colleges are represented with a non-negative integer mapping $l: C \rightarrow \mathbb{N}$. An applicant $a_{i}$ is admitted and allocated to a college $c_{u}$ if she achieves the score-limit at college $c_{u}$, and that is the first such place in her list, i.e. when $s_{u}^{i} \geq l\left(c_{u}\right)$, and $s_{v}^{i}<l\left(c_{v}\right)$ for every college $c_{v}$ such that $c_{v}>_{i} c_{u}$.

If the score-limits $l$ imply that applicant $a_{i}$ is allocated to college $c_{u}$, then we set the Boolean variable $x_{u}^{i}(l)=1$, and 0 otherwise. Let $x_{u}(l)=\sum_{i} x_{u}^{i}(l)$ be the number of applicants allocated to $c_{u}$ under score-limits $l$.

Furthermore, let $l^{u, t}$ be defined as follows: $l^{u, t}\left(c_{u}\right)=l\left(c_{u}\right)+t$ and $l^{u, t}\left(c_{v}\right)=l\left(c_{v}\right)$ for every $v \neq u$. That is, we increase the score-limit of college $c_{u}$ by $t$ (or decrease it if $t$ is negative), but we leave the other score-limits unchanged.

To introduce the $\mathrm{H}$-stable score-limits, first we define the corresponding feasibility notions. Score-limits $l$ are $H$-feasible if $x_{u}(l) \leq q_{u}$ for every college $c_{u} \in C$. That is, the number of applicants may not exceed the quota at any college. This means that the 
group of students with next lower scores, with whom the quota would be exceeded, are all rejected. Score-limits $l$ are $L$-feasible if for every college $c_{u} \in C$ such that $x_{u}(l) \geq q_{u}$ it must be the case that $x_{u}\left(l^{u, 1}\right)<q$. So the quotas may be exceeded at any college, but only with the worst group of students who are admitted there with equal scores.

We say that score-limits $l$ are $H$-stable (resp. L-stable) if $l$ are H-feasible (L-feasible) and for each college $c_{u}$ either $l\left(c_{u}\right)=0$ or $l^{u,-1}$ are not H-feasible (resp. L-feasible). Thus H-stability means that we cannot decrease the score-limit of any college without violating its quota assuming that the others do not change their limits. L-stability means that no college $c_{u}$ can admit a student if at least $q_{u}$ of its current assignees have a higher score, but otherwise the score limits must be as low as possible. H-stability is the concept that is currently applied in the Hungarian higher education matching scheme.

We note that in case of no tie (i.e. every pair of applicants have different scores at each college), then the two feasibility and stability conditions are the same and they are both equivalent to the original stability concept defined by Gale and Shapley. The correspondence between stable score-limits and stable matchings in case of strict preferences was first observed by Balinski and Sönmez [5] in relation with the Turkish college admissions scheme (where ties do not occur due to a tie-breaking strategy based on the age of the applicants). Furthermore Azevedo and Leshno [2] have also used this observation in a general college admissions model involving a continuum number of students.

\section{Stable score-limit algorithms}

Both the H-stable and L-stable score-limit algorithms are natural extensions of the GaleShapley algorithm. The only difference is that now, the colleges cannot necessarily select exactly as many of the best applicants as their quotas allow, since the applicants may have equal scores. If the scores of the applicants are all different at each college then these algorithms are equivalent to the original one. In this section we will present the applicant-proposing and the college-proposing score-limit algorithms. For simplicity we describe these algorithms with regard to the H-stability concepts only and we add some information about the L-stable versions in brackets whenever they differ from the H-stable versions.

\section{College-oriented algorithms:}

In the first stage of the algorithm, let us set the score-limit at each college independently to be the lowest value such that, when all applicants are considered, the number of applicants offered places does not exceed its quota (resp. may exceed the quota but only if without the last tie of these students the quota is unfilled). Let us denote these score-limits by $l_{1}$. Obviously, there can be some applicants who are offered places by several colleges. These applicants keep their best offer, and reject all the less preferred ones. Moreover, their less preferred applications are withdrawn, which means that less preferred colleges remove those applicants from the lists of considered applicants.

In the subsequent stages, the colleges check whether their score-limits can be further decreased, since some of their offers may have been rejected in the previous stage, hence they look for new students to fill the empty places. So each college sets its score-limit independently to be the least possible that keeps the solution $\mathrm{H}$-feasible (resp. L-feasible) considering their actual applications. If an applicant gets an offer from a new, better college, then she accepts the best offer, at least temporarily, and rejects or withdraws her other, less preferred applications.

Formally, let $l_{k}$ be the score-limit after the $k$-th stage. In the subsequent stage, at 
each college $c_{u}$, the largest integer $t_{u}$ is chosen, such that $t_{u} \leq l_{k}\left(c_{u}\right)$ and $x_{u}\left(l_{k}^{u,-t_{u}}\right) \leq q_{u}$ (resp. if $x_{u}\left(l_{k}^{u,-t_{u}}\right) \geq q_{u}$ then $\left.x_{u}\left(l_{k}^{u,-t_{u}+1}\right)<q_{u}\right)$. That is, by decreasing its score-limit by the largest score $t_{u}$ that keeps the solution $\mathrm{H}$-feasible (resp. L-feasible), i.e., where the number of applicants offered a place by $c_{u}$ does not exceed its quota (resp. may exceed the quota but only if without the last tie of these students the quota is unfilled), by supposing that all other score-limits remained the same. For each college $c_{u}$ let $l_{k+1}\left(c_{u}\right):=l_{k}^{u,-t_{u}}\left(c_{u}\right)$ be the new score-limit. Again, some applicants can be offered a place by more than one college, so $x_{u}\left(l_{k+1}\right) \leq x_{u}\left(l_{k}^{u,-t_{u}}\right)$. Obviously, the new score-limits remain feasible.

Finally, if no college needs to decrease its score-limit then the algorithm stops. The H-stability (resp. L-stability) of the final score-limits is obvious by definition. Let us denote the corresponding solutions of the H-stable and L-stable versions by $l_{C}^{H}$ and $l_{C}^{L}$, respectively.

\section{Applicant-oriented algorithms:}

Let each applicant propose to her first choice in her list. If a college receives more applications than its quota, then let its score-limit be the lowest value such that the number of provisionally accepted applicants does not exceed its quota (resp. may exceed the quota but only if without the last tie of these students the quota is unfilled). We set the other score-limits to be 0 .

Let the score-limits after the $k$-th stage be $l_{k}$. If an applicant has been rejected in the $k$-th stage, then let her apply to the subsequent college in her list, say $c_{u}$, where she achieves the actual score-limit $l_{k}\left(c_{u}\right)$, if there remains such a college in her list. Some colleges may receive new proposals, so if the number of provisionally accepted applicants exceeds the quota at a college (resp. exceeds the quota and without the last tie of these students the quota is still filled), then it sets a new, higher score-limit $l_{k+1}\left(c_{u}\right)$.

Again, for each such college $c_{u}$, this is the lowest score-limit such that the number of applicants offered a place by $c_{u}$ does not exceed its quota (resp. may exceed the quota but only if without the last tie of these students the quota is unfilled), by supposing that all other score-limits remained the same. This means that $c_{u}$ rejects all those applicants that do not achieve this new limit.

The algorithm stops if there is no new application. The final score-limits are obviously $\mathrm{H}$-feasible (resp. L-feasible). The solution is also H-stable (resp. L-stable), because after a score-limit has increased for the last time at a college, the rejected applicants move on to less preferred applications during the algorithm. So if the score-limit in the final solution were decreased by one for this college, then these applicants would accept the offer, and the solution would not remain H-feasible (resp. L-feasible). Let us denote the corresponding solutions by the $\mathrm{H}$-stable and L-stable applicant-oriented versions by $l_{A}^{H}$ and $l_{A}^{L}$, respectively. The following result is therefore immediate.

Theorem 3.1. The score-limits $l_{C}^{H}$ and $l_{C}^{L}$ obtained by the college-oriented score-limit algorithms are $H$-stable and $L$-stable, respectively. The score-limits $l_{A}^{H}$ and $l_{A}^{L}$ obtained by the applicant-oriented score-limit algorithms are H-stable and L-stable, respectively.

\section{Optimality of the outputs}

It is easy to give an example to show that not only some applicants can be admitted by preferred places in $l_{A}^{H}$ as compared to $l_{C}^{H}$, but the number of admitted applicants can also be larger in $l_{A}^{H}$ (and the same applies for the L-stable setting). We say that score-limits $l$ are better than $l_{*}$ for the applicants if $l \leq l_{*}$, i.e., if $l\left(c_{u}\right) \leq l_{*}\left(c_{u}\right)$ for every college 
$c_{u}$. In this case every applicant is admitted to the same or to a preferred college under score-limits $l$ than under $l_{*}$.

Theorem 4.1. Given a college admission problem with scores, $l_{C}^{H}$ are the worst possible and $l_{A}^{H}$ are the best possible $H$-stable score-limits for the applicants, i.e. for any H-stable score-limits $l, l_{A}^{H} \leq l \leq l_{C}^{H}$ holds.

Proof. Suppose first for a contradiction that there exists a $\mathrm{H}$-stable score-limit $l_{*}$ and a college $c_{u}$ such that $l_{*}\left(c_{u}\right)>l_{C}^{H}\left(c_{u}\right)$. During the college-oriented algorithm there must be two consecutive stages with score-limits $l_{k}$ and $l_{k+1}$, such that $l_{*} \leq l_{k}$ and $l_{*}\left(c_{u}\right)>l_{k+1}\left(c_{u}\right)$ for some college $c_{u}$.

Obviously, $l_{k}^{u,-t_{u}}\left(c_{u}\right)=l_{k+1}\left(c_{u}\right)$ by definition. Also, $x_{u}\left(l_{k}^{u,-t_{u}}\right) \leq q_{u}<x_{u}\left(l_{*}^{u,-1}\right)$, where the first inequality holds by definition of $t_{u}$, as we choose the new limit for college $c_{u}$ such a way that the number of temporarily admitted applicants does not exceed its quota. The second inequality holds by the H-stability of $l_{*}$. So there must be an applicant, say $a_{1}$, who is admitted to $c_{u}$ at $l_{*}^{u,-1}$ but not admitted to $c_{u}$ at $l_{k}^{u,-t_{u}}$.

On the other hand, the indirect assumption implies that $l_{k}^{u,-t_{u}}\left(c_{u}\right)=l_{k+1}\left(c_{u}\right) \leq$ $l_{*}\left(c_{u}\right)-1=l_{*}^{u,-1}\left(c_{u}\right)$. Applicant $a_{1}$ has a score of at least $l_{k}^{u,-t_{u}}\left(c_{u}\right)$, which is enough to be accepted to $c_{u}$, so she must be admitted to some college $c_{v}$ under $l_{k}^{u,-t_{u}}\left(c_{u}\right)$ which is preferred to $c_{u}$. Obviously $a_{1}$ must be also admitted to $c_{v}$ under $l_{k}$. But the H-stability of $l_{*}$ implies that $l_{*}\left(c_{v}\right)>l_{k}\left(c_{v}\right)$, a contradiction.

To prove the other inequality, we suppose for a contradiction that there exists H-stable score-limits $l_{*}$ and a college $c_{u}$ such that $l_{*}\left(c_{u}\right)<l_{A}^{H}\left(c_{u}\right)$. During the applicant-oriented algorithm there must be two consecutive stages with score-limits $l_{k}$ and $l_{k+1}$, such that $l_{*} \geq l_{k}$ and $l_{*}\left(c_{u}\right)<l_{k+1}\left(c_{u}\right)$ for some college $c_{u}$. At this moment, the reason for the incrementation is that more than $q_{u}$ students are applying for $c_{u}$ with a score of at least $l_{*}\left(c_{u}\right)$. This implies that one of these students, say $a_{i}$, is not admitted to $c_{u}$ under $l_{*}$ (however she has a score of at least $l_{*}\left(c_{u}\right)$ there). So, by the H-stability of $l_{*}$, she must be admitted to a preferred college, say $c_{v}$ under $l_{*}$. Consequently, $a_{i}$ must have been rejected by $c_{v}$ in a previous stage of the algorithm, and that is possible only if $l_{*}\left(c_{v}\right)<l_{k}\left(c_{v}\right)$, a contradiction.

Theorem 4.2. Given a college admission problem with scores, $l_{C}^{L}$ are the worst possible and $l_{A}^{L}$ are the best possible L-stable score-limits for the applicants, i.e. for any L-stable score-limits $l, l_{A}^{L} \leq l \leq l_{C}^{L}$ holds.

Proof. Suppose first for a contradiction that there exist stable score-limits $l_{*}$ and a college $c_{u}$ such that $l_{*}\left(c_{u}\right)>l_{C}^{L}\left(c_{u}\right)$. During the college-oriented algorithm there must be two consecutive stages with score-limits $l_{k}$ and $l_{k+1}$, such that $l_{*} \leq l_{k}$ and $l_{*}\left(c_{u}\right)>l_{k+1}\left(c_{u}\right)$ for some college $c_{u}$.

This assumptions imply that $x_{u}\left(l_{k}^{u,-t_{u}+1}\right)<q_{u} \leq x_{u}\left(l_{*}\right)$. Here, the first inequality holds by the L-feasibility of $l_{k+1}$, and the second inequality by the L-stability of $l_{*}$. At the same time, by our assumption, $l_{*}\left(c_{u}\right)>l_{k+1}\left(c_{u}\right)$, so $l_{*}\left(c_{u}\right) \geq l_{k+1}\left(c_{u}\right)+1=l_{k}^{u,-t_{u}+1}\left(c_{u}\right)$.

From the two above statements it follows that there must be an applicant, say $a_{1}$, who has a score $s_{u}\left(a_{1}\right) \geq l_{*}\left(c_{u}\right)$ and is admitted to $c_{u}$ under $l_{*}$, but is not admitted to $c_{u}$ under $l_{k}^{u,-t_{u}+1}$. So $a_{1}$ must have a seat at some college $c_{v}$ under $l_{k}^{u,-t_{u}+1}$ such that $c_{v}>_{a_{1}} c_{u}$. Obviously, $a_{1}$ is also admitted to $c_{v}$ under $l_{k}$. But $a_{1}$ is not admitted to $c_{v}$ under $l_{*}$, therefore $l_{k}\left(c_{v}\right)<l_{*}\left(c_{v}\right)$, a contradiction.

To prove the other inequality, we suppose for a contradiction that there exist stable score-limits $l_{*}$ and a college $c_{u}$ such that $l_{*}\left(c_{u}\right)<l_{A}^{L}\left(c_{u}\right)$. During the applicant-oriented algorithm there must be two consecutive stages with score-limits $l_{k}$ and $l_{k+1}$, such that $l_{*} \geq l_{k}$ and $l_{*}\left(c_{u}\right)<l_{k+1}\left(c_{u}\right)$ for some college $c_{u}$. 
At this moment, the reason for the incrementation is that more than $q_{u}$ students are applying for $c_{u}$ with score at least $l_{*}(u)$, and $c_{u}$ can choose a new score-limit $l_{k+1}\left(c_{u}\right)=$ $l_{k}^{u,-t_{u}}\left(c_{u}\right)$, where $t_{u}>l_{*}\left(c_{u}\right)-l_{k}\left(c_{u}\right)$.

This implies that one of those students, who are admitted by $c_{u}$ under $l_{k+1}$, say $a_{1}$, is not admitted to $c_{u}$ under $l_{*}$. However she has a score higher than score-limit $l_{*}\left(c_{u}\right)$ there. So, by the L-stability of $l_{*}$, she must be admitted to a preferred college, say $c_{v}$, under $l_{*}$. Consequently, in the applicant-proposing procedure $a_{1}$ must have been rejected by $c_{v}$ at some previous stage, and that is possible only if $l_{*}\left(c_{v}\right)<l_{k}\left(c_{v}\right)$, a contradiction.

\section{General arguments with choice functions}

As we mentioned in the Introduction, our results presented in Sections 3 and 4 are deducible from some general theorems on substitutable choice functions by Kelso and Crawford [18] and Roth [23], as Fleiner and Jankó [13] pointed out. The selection of the colleges can be described by their choice functions. For a college $u$ and a set of applicants $X$, let $C h_{u}(X)$ denote the set of selected applicants. A choice function $C h_{u}$ is substitutable (or comonotone) if $X \subseteq Y$ implies $\left(X \backslash C h_{u}(X)\right) \subseteq\left(Y \backslash C h_{u}(Y)\right)$, which means that the set of applicants rejected from a set $X$ must be also rejected from its superset $Y$. The choice functions of the colleges satisfy this condition with respect to both the L-stable and H-stable score limits. That is, if the colleges consider a larger set of applications then no application that has been rejected can turn to be acceptable in any case. Kelso and Crawford [18] showed that if the choice functions are substitutable on both sides of a many-to-one markets then there always exists a stable matching, moreover there is one stable matching that is optimal for the colleges. Roth [23] showed the existence of an applicant-optimal matching for this model (and also for the more general many-to-many case).

Furthermore, Fleiner and Jankó [13] gave new results on the structure of stable matchings that applies for L-stable and H-stable score limits as well. They noticed that the choice function of the colleges under L-stability satisfy the path-independence property, that is for any set of applicants $X \subseteq Y, C h_{u}(Y) \subseteq X \subseteq Y$ implies $C h_{u}(X)=C h_{u}(Y)$. Therefore the theorem of Blair [9] implies that the set of stable matchings corresponding to L-stable score-limits forms a lattice. However, the path-independence property does not hold for the choice functions related to H-stable score-limits. Yet, the stable matchings corresponding to H-stable score-limits form a lattice, as Fleiner and Jankó proved with the use of new concept, called four-stability.

\section{Comparison of the $\mathrm{H}$-stable and L-stable versions}

Intuitively it seems that the L-stable version of the algorithm is more applicant-friendly than the H-stable version. It turns out that we can prove the following result.

Theorem 5.1. The score-limits obtained in the L-stable version of the applicants-oriented procedure are always equal or lower than the score-limits obtained in the H-stable version of the applicant-oriented procedure: i.e. $l_{A}^{L} \leq l_{A}^{H}$.

Proof. Part I. Some colleges may have number of admitted students less than or equal to their quota under $l_{A}^{H}$, i.e. $q_{u}-x_{u}\left(l_{A}^{H}\right) \geq 0$. Each college $c_{u}$ has a "waiting" list of applicants, who would prefer to be admitted to $c_{u}$ rather than to their currently assigned colleges.

Let us apply some random tie-breaking to the original preference relation of the colleges. Each applicant $a_{i}$ will get a new score $p_{u}^{i} \geq s_{u}^{i}$ such that no two applicants will have 
the same score at any college. Moreover, the new scores satisfy the following condition: if $s_{u}^{j}<s_{u}^{i}$, then $p_{u}^{j}<s_{u}^{i}$. These $p_{u}^{i}$ scores are positive real numbers. For example, if there are three applicants with scores $s_{u}^{1}=s_{u}^{2}=1, s_{u}^{3}=2$, the new scores might be $p_{u}^{1}=1$, $p_{u}^{2}=1.5, p_{u}^{3}=2$.

After that the following procedure is organized. If the number of applicants on $c_{u}$ college's waiting list is more than the number of empty seats then college $c_{u}$ sets it's new score-limit $m_{A}^{H}\left(c_{u}\right) \leq l_{A}^{H}\left(c_{u}\right)$ equal to the score $p_{u}^{i}$ of the last admitted applicant in its waiting list. Otherwise let $m_{A}^{H}\left(c_{u}\right)=0$. Note that the new score-limits $m_{A}^{H}$ are nonnegative real numbers. This means that each college make offers to applicants from its waiting list who fit the new score-limit.

Some applicants may receive more than one proposal. Each applicant accepts one, from the most preferred college, and rejects the others. If there remain any empty seat in colleges then the second step is organized in the same manner and so on. Thus essentially we run a college-proposing deferred-acceptance procedure with regard to the new scores. At the end of this procedure some new score-limits $m^{R}$ are achieved such that $m^{R} \leq l_{A}^{H}$ by construction. These new score-limits $m^{R}$ and the corresponding matching $\mu^{R}$ are stable (in the Gale-Shapley sense) according to new strict preferences of colleges, also by construction.

Part II. For the strict preference profile and corresponding scores $p_{u}^{i}$ from Part I we can organize applicant-proposing deferred acceptance procedure (which is, in case of strict preferences, equivalent to both the H-stable and L-stable applicant-oriented algorithms). The resulting matching $\mu_{A}^{R}$ is, of course, stable under strict preferences. Furthermore, we can define score-limits $m_{A}^{R}$ that are equal to the score of the last accepted applicant if college has no empty seats and to 0 otherwise. These score-limits $m_{A}^{R}$ must be the lowest among all stable score-limits by the optimality theorem of Gale and Shapley. Therefore $m_{A}^{R} \leq m^{R}$ in particular.

Part III. Now we deal with $m_{A}^{R}$ score-limits. Let us get back to the original weak order preferences of the colleges and corresponding applicants' scores $s_{u}^{i}$. For each college with $x_{u}\left(m_{A}^{R}\right)=q_{u}$ we can construct a "waiting" list of applicants, who prefer college $c_{u}$ to their current matches under $m_{A}^{R}$.

Let us now apply the L-feasibility concept. At the first stage each college sets it's new score-limit $l_{A}^{R}\left(c_{u}\right) \leq m_{A}^{R}\left(c_{u}\right)$, that is the largest value, which allows to admit equal or more than the quota under weak order preferences as L-feasibility prescribes. For example, if there are two applicants with the same score $s_{u}^{i}$, such that one of them is admitted to $c_{u}$ under $m_{A}^{R}$ and the other is on the waiting list then we have to 'treat them equally', so we should lower the score-limit. Each college makes offers to these additional applicants.

Some applicants may receive more than one offer from colleges; in this case each applicant chooses the most preferred college. After that if there is any college with number of admitted applicants less than its quota then a new round starts. Each college chooses new, lower, L-feasible limit, and so on. That is we run the college-proposing score-limit procedure under L-stability. At the end, some new score-limits $l^{L}$ are achieved such that $l^{L} \leq m_{A}^{R}$ by construction. These new score limits are L-feasible and L-stable, obviously.

Part IV. For each L-stable score-limit $l^{L}$ we know that $l_{A}^{L} \leq l^{L}$ from Theorem 4.2, where $l_{A}^{L}$ are stable score-limits obtained by the L-stable applicant-oriented algorithm.

Now we can construct the following inequalities: $l_{A}^{L} \leq l^{L} \leq m_{A}^{R} \leq m^{R} \leq l_{A}^{H}$. So we can conclude that for any college admissions problem with score-limits the outcome by the L-stable applicant-oriented algorithm is better for the applicants (i.e. yields lower score-limits) than the outcome of the H-stable applicant-oriented algorithm. 
Theorem 5.2. The score-limits obtained in the L-stable version of the college-oriented procedure are always equal or lower than the score-limits obtained in the H-stable version of the college-oriented procedure: i.e. $l_{C}^{L} \leq l_{C}^{H}$.

Proof. Part I. Let us consider the $l_{C}^{L}$ score-limits. Some colleges may have number of admitted students more than or equal to their quota, $x_{u}\left(l_{C}^{L}\right) \geq q_{u}$.

Let us apply a random tie-breaking to the original preference relation of the colleges. Each applicant $a_{i}$ gets a new score $p_{u}^{i} \geq s_{u}^{i}$ such that no two applicants have the same score at any college, and these new scores do not contradict with the original ordering. Moreover, if $s_{u}^{j}<s_{u}^{i}$, then $p_{u}^{j}<s_{u}^{i}$. These $p_{u}^{i}$ scores are positive real numbers.

After that the following procedure is organized. At the first stage each college sets its new score-limit $m_{C}^{L}\left(c_{u}\right) \geq l_{C}^{L}\left(c_{u}\right)$ such that according to the new scores $p_{u}^{i}$ the number of applicants who fit this score-limit would be exactly $q_{u}$. The new score-limits $m_{C}^{L}$ are non-negative real numbers. Let $m_{C}^{L}\left(c_{u}\right)$ be equal to 0 if the number of students admitted to $c_{u}$ is less than $c_{u}$ 's quota and otherwise let $m_{C}^{L}\left(c_{u}\right)$ be equal to the lowest score $p_{u}^{i}$ of any admitted student.

Some applicants are rejected from colleges they were assigned under $l_{C}^{L}$. Each rejected applicant then applies to the subsequent college in her list. Colleges receive new applications and, if necessary, raise the limits so that number of accepted applicants are equal to their quota. Some new applicants may be rejected, so a second round is organized in the same manner and so on. Thus we run an applicant-proposing deferred-acceptance procedure with respect to the perturbed strict preferences. At the end, some new scorelimits $m^{R}$ are obtained such that $m^{R} \geq l_{C}^{L}$ by construction. These new score-limits are stable (in the Gale-Shapley sense) according to the new strict preferences of colleges by construction.

Part II. For strict preference profile and corresponding scores $p_{u}^{i}$ from Part I we can organize a college-oriented deferred-acceptance procedure. The resulting score-limits $m_{C}^{R}$ are, of course, stable according to these strict preferences. Furthermore, the corresponding score-limits must be the lowest among all stable score-limits by the optimality theorem of Gale and Shapley [14], so $m_{C}^{R} \geq m^{R}$.

Part III. Now we deal with $m_{C}^{R}$ score-limits. For each college $c_{u}, x_{u}\left(m_{C}^{R}\right) \leq q_{u}$ holds under $m_{C}^{R}$. Each college $c_{u}$ with number of assigned students lower than its quota has score-limit $m_{C}^{R}\left(c_{u}\right)=0$. Now we get back to the original weak order preferences of the colleges and original applicants' scores $s_{u}^{i}$.

Let us now apply the H-feasibility concept. For each college we can construct a list of applicants, who prefer college $c_{u}$ to their current matches under $m_{C}^{R}$. After that the following deferred acceptance procedure is organized. At the first stage each college sets it's new score-limit $l_{C}^{R}\left(c_{u}\right) \geq m_{C}^{R}\left(c_{u}\right)$ that is the lowest value, which allows to admit equal or less than the quota under weak order preferences as $\mathrm{H}$-feasibility prescribes. Therefore some colleges may reject applicants. Each rejected applicant applies to the next college in her list. Colleges receive new applications and, if necessary, raise their score-limits in such a way that the number of accepted applicants is less than or equal to their quota. Some applicants may be rejected and a second round is organized in the same manner and so on. Thus we run an applicant-proposing deferred-acceptance procedure with regard to H-stability. At the end, each applicant is either accepted to some college or rejected by all acceptable colleges. Some new score-limits $l^{H}$ are achieved such that $l^{H} \geq m_{C}^{R}$ by construction. These new score-limits are $\mathrm{H}$-feasible and H-stable, obviously.

Part IV. For each H-stable score-limit $l^{H}$ we know that $l_{C}^{H} \geq l^{H}$ from theorem 4.1, where $l_{C}^{H}$ is a H-stable score-limit obtained by the applicant-oriented score-limit algorithm.

Now we can construct the following inequalities: $l_{C}^{L} \leq m^{R} \leq m_{C}^{R} \leq l^{H} \leq l_{C}^{H}$. So we can conclude that for any college admissions problem with score-limits the outcome 
by the L-stable college-oriented algorithm is better for the applicants (i.e. yields lower score-limits) than the outcome of the H-stable college-oriented algorithm.

Corollary 5.1. Applicant-optimal $H$-stable and L-stable score-limits $\left(l_{A}^{H}\right.$ and $\left.l_{A}^{L}\right)$ are upper and lower boundaries, respectively, for score-limits under any applicant-optimal stable matching with random tie-breaking. Similar statement applies for the applicant-pessimal score-limits $\left(l_{C}^{H}\right.$ and $\left.l_{C}^{L}\right)$.

Finally, we note that the proofs of Theorem 5.1 and 5.2 can be naturally extended to get an implication that is slightly more general than the above Corollary. Suppose that the applicants could be strictly ranked by the colleges with exact scores $e$, but the scoring method used in the scheme is not fine enough, which leads to rounded scores $s$ with ties. We say that scores $f$ are refinements of scores $s$ if $f$ and $s$ are not the same, and for any pair of applicants $a_{i}$ and $a_{j}$ and college $c_{u}, s_{u}^{i} \leq f_{u}^{i} \leq e_{u}^{i}, s_{u}^{i}<s_{u}^{j}$ implies $f_{u}^{i}<s_{u}^{j}$ and $f_{u}^{i}<f_{u}^{j}$ implies $e_{u}^{i}<f_{u}^{j}$. Intuitively, if there exist exact scores $e$ then a scoring method is finer than another one if it produce scores closer to $e .^{6}$

Corollary 5.2. Suppose that the applicants can be ranked with exact scores $e$ by the colleges, and the corresponding applicant-optimal stable score-limits are $l_{A}(e)$. Let $s$ and $f$ be scores such that e are refinements of $f$ and $f$ are refinements of $s$. Let $l_{A}^{H}(s)$ and $l_{A}^{L}(s)$ denote the applicant-optimal stable score-limits for scores $s$ with respect to the H-stable and L-stable concepts. Then $l_{A}^{L}(s) \leq l_{A}^{L}(f) \leq l_{A}(e) \leq l_{A}^{H}(f) \leq l_{A}^{H}(s)$ holds.

\section{$6 \quad$ Strategic issues}

Here we give two examples showing that neither of the above described score-limit algorithms is strategy-proof. The manipulability from the applicants' side is only interesting in the case of applicant-oriented algorithms, as the applicants may successfully manipulate the college-oriented versions even for strict preferences (i.e., for scores with no ties). Therefore we only consider the applicant-oriented versions in the examples below.

Example 1. Suppose that we have two colleges, $c_{u}$ and $c_{v}$ with one seat in each of them, and two applicants $s_{1}$ and $s_{2}$ applying to both $c_{u}$ and $c_{v}$ with a preference towards $c_{u}$ and with equal scores at both places. So the preference list of the colleges and students are as follows.

$$
\begin{array}{ll}
a_{1}: c_{u}, c_{v} & c_{u}:\left(a_{1}, a_{2}\right) \\
a_{2}: c_{u}, c_{v} & c_{v}:\left(a_{1}, a_{2}\right)
\end{array}
$$

Figure 1: An example for the manipulability of the H-stable applicant-proposing algorithm

Here the only H-stable solution is the empty matching (i.e., score-limits higher than the scores of $a_{1}$ and $a_{2}$ at both colleges). However, if either of the students, say $a_{1}$ withdraws her application at $c_{u}$ then the unique $H$-stable solution (under falsified preferences) is matching where $a_{1}$ is allocated to $c_{v}$ and $a_{2}$ is allocated to $c_{u}$. So the manipulator (and actually the other student also) would improve.

\footnotetext{
${ }^{6}$ In Hungary the scoring method became finer in 2007. Until 2007 each written exam with a maximum score of 100 had been rounded to an integer score between 0 and 15. Since 2007 the exact score of these written exams are considered when calculating the final scores of the students. As a result the maximum score increased from 144 to 480 and the ties in the rankings of the colleges became less common and between a smaller number of students.
} 
The following example is essentially the same as the one that Hatfield and Milgrom [15] constructed in a different setting but for a similar purpose.

Example 2. Suppose that we have two colleges, $c_{u}$ and $c_{v}$ with one seat in each of them, and three applicants $a_{1}, a_{2}$ and $a_{3}$ applying to both $c_{u}$ and $c_{v}$ with the following scores, $s_{u}^{1}=1, s_{u}^{2}=1, s_{u}^{3}=2, s_{v}^{1}=3, s_{v}^{2}=2$ and $s_{v}^{3}=1$. These can be described equivalently with the preference lists below.

$$
\begin{array}{ll}
a_{1}: c_{u}, c_{v} & c_{u}: a_{3},\left(a_{1}, a_{2}\right) \\
a_{2}: c_{v}, c_{u} & c_{v}: a_{1}, a_{2}, a_{3} \\
a_{3}: c_{v}, c_{u} &
\end{array}
$$

Figure 2: An example for the manipulability of the L-stable applicant-proposing algorithm

Here the only L-stable solution is the matching $\left\{\left(a_{1}, c_{v}\right),\left(a_{3}, c_{u}\right)\right\}$ (i.e., with scorelimits $l\left(c_{u}\right)=2$ and $\left.l\left(c_{v}\right)=3\right)$. However, if $a_{2}$ were to reverse her preferences with regard to the two colleges then the L-stable applicant-oriented algorithm would produce the matching $\left\{\left(a_{1}, c_{u}\right),\left(a_{2}, c_{u}\right),\left(a_{3}, c_{v}\right)\right\}$, where the manipulator (and actually both the two other applicants) would improve.

\section{$7 \quad$ Further notes}

Regarding the Hungarian application, we would like to conduct an experiment with real data and compute the four possible extreme stable score-limits, namely the applicantoptimal and applicant-pessimal score-limits under H-stability and L-stability. It would also be interesting to see how these concepts can be used in other settings, e.g. what could be the corresponding solutions for the Boston and New York high school matching programs.

Regarding the theoretical problems, we would like to investigate whether there is any structure behind the H-stable and L-stable score-limits. It would also be worth studying further the relation of solutions satisfying equal treatment policy and those produced by tie-breaking strategies.

Finally, some manipulable stable matching mechanisms turned out to be incentive compatible in large markets $[25,16,20]$. Our question is whether either of the applicantoriented stable score-limit algorithms is approximately strategyproof in large markets.

\section{Acknowledgements}

We would like to thank to Gábor Varjasy, the representative of Educatio kht (the nonprofit governmental organization which runs the higher education admission scheme in Hungary). Further we acknowledge Tamás Fleiner, Rob Irving and two referees for their useful comments and we also thank Jordi Masso and Antonio Romero-Medina for their help in understanding the Spanish higher education admissions system. Finally we would like to thank the participants of the Frontiers of Market Design: Matching Markets Conference, the 8th Spain-Italy-Netherlands Meeting on Game Theory, the Fourth Congress of the Game Theory Society, the 11th Meeting of Society of Social Choice and Welfare and the Fourth International Workshop on Computational Social Choice, for giving useful feedback. 


\section{References}

[1] A. Abdulkadiroğlu, P.A. Pathak, and A.E. Roth. Strategy-proofness versus efficiency in matching with indifferences: Redesigning the NYC high school match. American Economic Review, 99(5):1954-1978, 2009.

[2] E. Azevedo and J. Leshno. A supply and demand framework for two-sided matching markets. working paper, 2011.

[3] A. Abdulkadiroğlu, P.A. Pathak, and A.E. Roth. The New York City high school match. American Economic Review, Papers and Proceedings, 95(2):364-367, 2005.

[4] A. Abdulkadiroğlu, P.A. Pathak, A.E. Roth, and T.Sönmez. The Boston public school match. American Economic Review, Papers and Proceedings, 95(2):368-371, 2005.

[5] M. Balinski and T. Sönmez. A tale of two mechanisms: Student placement. Journal of Economic Theory, 84(1):73-94, 1999.

[6] P. Biró. Student admissions in Hungary as Gale and Shapley envisaged. Technical Report no. TR-2008-291 of the Computing Science Department of Glasgow University, 2008.

[7] P. Biró, T. Fleiner, R.W. Irving, and D.F. Manlove. College admissions with lower and common quotas. Theoretical Computer Science, 411:3136-3153, 2010.

[8] P. Biró, F. Klijn. Matching with Couples: a Multidisciplinary Survey. To appear in International Game Theory Review, 2012.

[9] C. Blair. The lattice structure of the set of stable marriages with multiple partners. Mathematics of Operations Research, 13(4):619-628, 1988.

[10] S. Braun, N. Dwenger, and D. Kübler. Telling the truth may not pay off: An empirical study of centralised university admission in Germany. 2010. The B.E. Journal of Economic Analysis and Policy, 10(1): Article 22.

[11] C. Calsamiglia, G. Haeringer, and F. Klijn. 'Constrained School Choice: An Experimental Study. American Economic Review, 100(4):1860-1874, 2010.

[12] A. Erdil and H. Erkin. What's the matter with tie-breaking? improving efficiency in school choice. American Economic Review, 98:669-689, 2008.

[13] T. Fleiner and Zs. Jankó. Choice function based two-sided markets: stability, lattice property and path independence. unpublished manuscript, 2012.

[14] D. Gale and L.S. Shapley. College Admissions and the Stability of Marriage. American Mathematical Monthly, 69(1):9-15, 1962.

[15] J.W. Hatfield and P.R. Milgrom. Matching with contracts. American Economic Review, 95(4):913-935, 2005.

[16] N. Immorlica and M. Mahdian. Marriage, Honesty, and Stability. In Proceedings of SODA 2005, 53-62, 2005.

[17] R.W. Irving and D.F. Manlove. Finding large stable matchings. ACM Journal of Experimental Algorithmics, 14, section 1, article 2, 30 page. 
[18] A.S. Kelso and V.P. Crawford. Job Matching, Coalition Formation, and Gross Substitutes. Econometrica, 50: 1483-1504, 1982.

[19] Knuth, D. E. Mariages Stables. Les Presses de L'Universite de Montreal, 1976.

[20] F. Kojima and P.A. Pathak. Incentives and Stability in Large Two-Sided Matching Markets. American Economic Review, 99:608-627, 2009.

[21] A. Romero-Medina. Implementation of stable solutions in a restricted matching market. Review of Economic Design, 3(2):137-147, 1998.

[22] A.E. Roth The Economics of Matching: Stability and Incentives. Mathematics of Operations Research, 7: 617-628, 1982.

[23] A.E. Roth Stability and Polarization of Interests in Job Matching. Econometrica, 52: 47-58, 1984.

[24] A.E. Roth. The evolution of the labor market for medical interns and residents: a case study in game theory. Journal of Political Economy, 6(4):991-1016, 1984.

[25] A.E. Roth and E. Peranson The Redesign of the Matching Market for American Physicians: Some Engineering Aspects of Economic Design. American Economic Review, 89:748-780, 1999.

[26] A. Westkamp. An analysis of the German university admissions system. Bonn Econ Discussion Papers, no bgse02_2012, 2012.

[27] H. Zhang. An Analysis of The Chinese College Admission System. PhD Thesis, University of Edinburgh, 2009.

[28] Y. Zhang. The determinants of National College Entrance Exam performance in China - with an analysis of private tutoring. PhD Thesis, Columbia University, 2011.

[29] Central Applications Office, Ireland website. www.cao.ie

[30] D. Kübler. University admission practices - Germany. matching-in-practice.eu, accessed on 23 May 2012

[31] P. Biró. University admission practices - Hungary. matching-in-practice.eu, accessed on 23 May 2012

[32] R. Irving. Matching practices for entry-labor markets - Scotland. matching-inpractice.eu, accessed on 23 May 2012

[33] Matching in Practice website. matching-in-practice.eu

[34] Al Roth's market designer blog. http://marketdesigner.blogspot.com

[35] UCAS website. http://www.ucas.com 